\title{
Correspondence
}

\section{Association between rheumatoid arthritis and chronic bronchial suppuration}

SIR, We have investigated the coexistence of rheumatoid arthritis (RA) and chronic bronchial suppuration (CBS) and concluded, in a report to be published shortly, that there is a true association between the diseases. ${ }^{1}$ As part of this study we found four patients with RA who had recurrent acute respiratory tract infections associated with hypogammaglobulinaemia. Therefore we read with great interest the report of So, Peskett, and Webster ${ }^{2}$ suggesting that gold can cause immunodeficiency.

Our four patients were hypogammaglobulinaemic when first investigated for their respiratory problems. All were IgM rheumatoid factor (IgMRF) negative at that time, but all had been IgMRF positive at some previous time. All had been on gold; one patient had normal immunoglobulin (Ig) levels prior to gold treatment, but baseline levels were not available in the other three.

A fifth patient with IgMRF positive RA presented with acute recurrent chest infections while on gold and was found to be hypogammaglobulinaemic. Gold therapy was stopped for lack of effect and Ig levels subsequently returned to normal, but the patient developed bronchiectasis.

The prior seropositivity of all five patients indicates that their arthritis could not itself be attributed to the hypogammaglobulinaemia.

We suggested that: (1) gold was implicated in the genesis of the hypogammaglobulineamia, (2) this could predispose the patient to the development of recurrent acute chest infections, and (3) chronic bronchial suppuration might develop subsequently.

The severity of chest symptoms in our study group was such that we concluded our report: 'If an inherent predisposition to infection in RA is exacerbated by disease-modifying drugs then risk factors for CBS should be identified before starting treatment with these drugs and immunoglobulins monitored regularly. A significant fall necessitates drug withdrawal.' Our own experience suggests however that such monitoring should not be confined to IgMRF negative patients.

Lastly, it appears that the first case described by So $e t$ al. had 'gold lung', which is rare ${ }^{3}$ and not previously reported to be associated with hypogammaglobulinaemia.

Department of Rheumatology, ANDREW BAMJI

Brook General Hospital,

Shooters Hill Road,

London SE18 4LW

Department of Medicine, Charing Cross Hospital,

NIGEL T. COOKE

Fulham Palace Road,

London W6 8RF
References

1 Bamji A N, Cooke N T. Rheumatoid arthritis and chronic bronchial suppuration. Scand $J$ Rheumatol in press.

2 So A K L, Peskett S A, Webster A D B. Hypogammaglobulinaemia associated with gold therapy. Ann Rheum Dis 1984; 43: 581-2.

3 Cooke N T, Bamji A N. Gold lung. Rheumatol Rehabil 1981; 20: $129-35$.

\section{Vertebral rim lesions in dorsolumbar spine}

SIR, We wish to reply to the letter from Professor B. Vernon-Roberts. ${ }^{1}$ Much as we value the work of Schmorl and Junghanns ${ }^{2}$ they do not describe vertebral rim lesions, and the concept of occult traumatic lesions of the anterior vertebral rim in the lumbar spine and dorsolumbar junction as a potential cause of idiopathic low back pain does not emerge from their writings. The same can be said of the paper by Vernon-Roberts and Pirie. ${ }^{3}$ Both references describe disc tears at length, including those running close to the rim, but mainly in relation to the pathogenesis of prolapse and osteophyte formation.

While we accept that tears occurring close to the vertebral rim may be due to trauma, the pathological hallmark of a vertebral rim lesion is traumatic damage to the bone. ${ }^{4}$ These bone lesions are not always associated with a tear in the adjacent annulus. When present the tear doesn't just occur near the rim as described by VernonRoberts and Pirie ${ }^{3}$ and Schmorl and Junghanns ${ }^{2}$ but runs down to the site of the vertebral bone damage. As bone is known to be innervated by pain fibres, these lesions may have a greater potential for causing pain than annular tears occurring alone.

Vernon-Roberts implies that tears occurring at the annular attachment cannot be seen on clinical radiographs. We do not agree. Fig. 1 is a clinical radiograph showing a translucency in the annulus which is accompanied by sclerosis in the underlying rim giving an overall appearance identical to the lesion shown in Fig. 2 of our paper. ${ }^{4} \mathrm{We}$ have used the term vacuum phenomenon in relation to the annular translucency because it describes a radiological appearance and we can see no reason why the term should be restricted to degenerative clefts occurring in the nucleus.

Department of Rheumatology

R. C. HILTON

Hope Hospital, J. BALL

Eccles Old Road,

Salford M6 8HD 


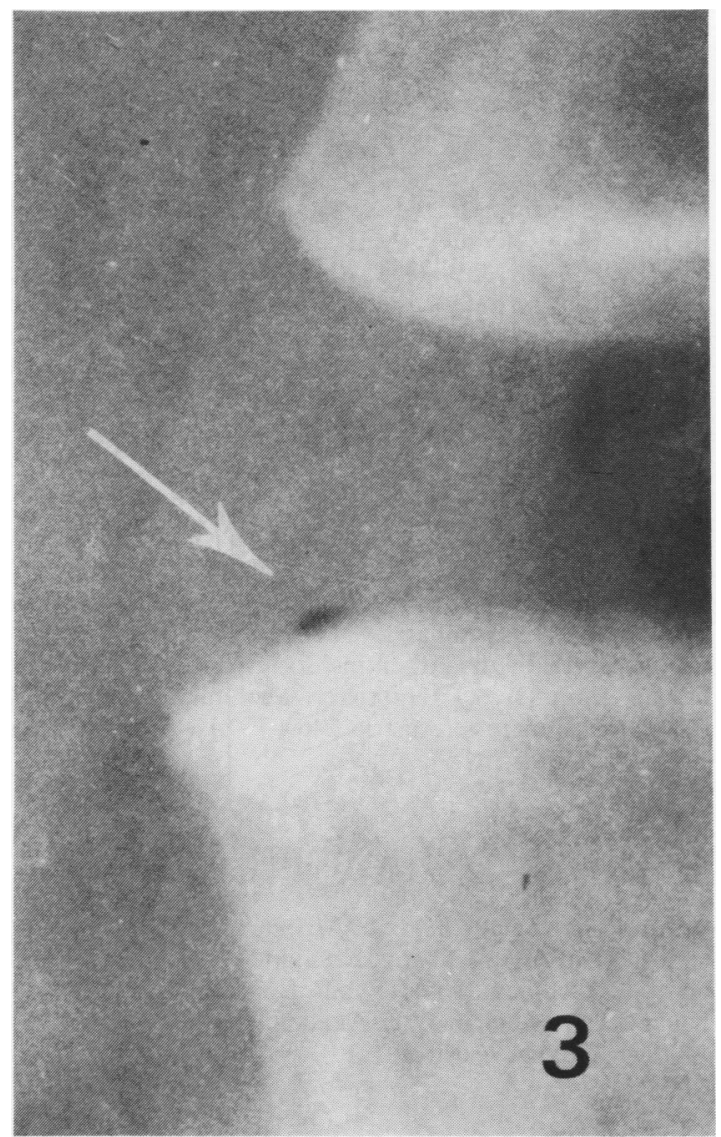

Clinical radiograph of a female aged 40 showing a rim lesion at the upper anterior corner of L3. There is localised sclerosis with an adjacent annular translucency indicating the presence of a tear (arrowed).

\section{References}

1 Vernon-Roberts B. Vertebral rim lesions in dorsolumbar spine. Ann Rheum Dis 1984; 43: 662.

2 Schmorl G, Junghanns $\mathrm{H}$. The human spine in health and disease. 2nd American ed. New York and London: Grune and Stratton, 1971.

3 Vernon-Roberts B, Pirie C. J. Degenerative changes in the intervertebral discs of the lumbar spine and their sequelae. Rheumatol Rehabil 1977; 16: 13-21.

4 Hilton R C, Ball J. Vertebral rim lesions in the dorsolumbar spine. Ann Rheum Dis 1984; 43: 302-7.

\section{Colchicine in systemic amyloidosis}

SIR, We read with interest the report by Scheinberg et al. ${ }^{1}$ of DMSO and colchicine therapy in amyloid disease. The beneficial effects of colchicine on systemic amyloidosis have been suggested by other investigators. ${ }^{2+}$ We also have investigated the effect of colchicine on patients with primary (five patients), reactive (three patients), and familial Mediterranean fever (FMF) related secondary amyloidosis (eight patients). ${ }^{5}$

At six months, 11 patients out of 16 revealed decreased proteinuria, increased serum albumin level, and overt clinical improvement. ${ }^{5}$ Twenty-four hour creatinine clearances were also increased in some degree. During this six-month period colchicine was found to be more beneficial for the patients with amyloidosis secondary to FMF, though two out of five cases with the primary form have also shown some improvement. However, follow-up of some of these patients to two years showed this beneficial effect of colchicine to be temporary even in FMF related patients. Most of them have shown both clinical and laboratory deterioration during the following months.

Therefore, our experience of long-term colchicine treatment for systemic amyloidosis is rather disappointing, and we suggest that the effect of the drug on amyloidosis needs further evaluation in a long-term and large-scale investigation.

Çukurova University,

E. AKOǦLU

Medical Faculty,

T. AKOĞLU

Department of Internal Medicine

E. ERKEN

Adana,

Turkey

\section{References}

1 Scheinberg M A, Pernambuco J C, Benson M D. DMSO and colchicine therapy in amyloid disease. Ann Rheum Dis 1984; 43: 421-3.

2 Shirahama T, Cohen A S. Blockage of amyloid induction by colchicine in an animal model. $J$ Exp Med 1974; 140: 1102-7.

3 Kedar I, Ravid M, Sohar E. Colchicine inhibition of casein induced amyloidosis in mice. Isr J Med Sci 1974; 10: 787-92.

4 Ravid M, Robson M, Kedar I. Prolonged colchicine treatment in four patients with amyloidosis. Ann Intern Med 1977; 27: 568-70.

5 Akoğlu E, Akoğlu T, Gürçay A, Turgan Ç, Çağlar S, Yasavul $\mathrm{U}$. The effect of colchicine on systemic amyloidosis. J Cukurova Medical Faculty 1982; 7: 48-55. 